\title{
Understanding of attentional suppression is incomplete without consideration of motivation and context
}

Andrew B. Leber

Department of Psychology, The Ohio State University, 225 Psychology Building, 1835 Neil Avenue, Columbus, OH, 43210. E-mail: leber.30@,osu.edu

\begin{abstract}
Luck, Gaspelin, Folk, Remington, and Theeuwes (2021) provide a valuable status report on our collective understanding of attentional capture, and they succeed at identifying common ground and articulating persisting points of discord. Here, I contribute two points. First, while there is limited evidence individuals can explicitly and proactively suppress salient distractors, investigations into this question have not always considered participants' motivation and strategy use. Second, in evaluating how attentional suppression is guided by past experience, or selection history, one must consider the significant role of associative learning, which is revealed via context-dependent phenomena.
\end{abstract}

The authors of the target article (Luck, Gaspelin, Folk, Remington \& Theeuwes, 2021) are to be commended for their attempts at both synthesizing a vast and nuanced literature and at bridging vast and nuanced theoretical divides. In a discipline of study that is often best known for its disagreements, it is refreshing to acknowledge and articulate what common ground exists. I will use this commentary to address two points.

\section{Why Would our Participants Use Explicit Proactive Suppression?}

One key area of remaining disagreement regards whether explicit, proactive suppression is possible, with Gaspelin \& Luck expressing skepticism and Folk \& Remington more enthusiastically embracing it. Theeuwes stakes the bold claim that proactive feature-based suppression - even of the implicit flavor has yet to be convincingly demonstrated. Each group raises thoughtful points, but the extant literature admittedly lacks the evidence to usher a resolution. I believe our failure to sufficiently consider motivation explains this lack of parsimony.

Discussion of motivation is largely absent from the target article, but researchers have pondered it for decades. Bacon and Egeth (1994) speculated that effort avoidance was at play when questioning why people would allow themselves to be distracted if they were otherwise capable of overriding it. Another way of thinking about this is, why would participants exert the effort needed to maintain a control state if such a state only saves a cumulative cost of capture of a few seconds across an entire experimental session (Egeth, Leonard, \& Leber, 2010)?

Consider the conditions researchers have placed on participants to demonstrate explicit, proactive suppression. First, on each trial, participants must process a cue telling them what color the distractor will be. Then, if the cue differs from the previous trial, they must update their control state to suppress the now to-be-ignored feature. During this procedure, participants are given feedback about their accuracy and occasionally about their speed, but not about whether they successfully achieved proactive suppression. Given the weak incentives and imprecise feedback, it may be unsurprising that participants decline to implement proactive suppression. Moreover, researchers typically examine session-averaged data, which may overlook periods of time in which participants are successful.

In many ways the debate over explicit proactive suppression is reminiscent of a classic debate in the task switching literature (Monsell, 2003). This debate centered around whether or not one can 
intentionally switch their task set in advance of the trial and avoid the costs associated with reactive switching. But one can ask not whether participants are capable of proactively switching but instead whether they want to do so. This question was addressed by Arrington and Logan (2004), who modified the conventional task switching procedure by allowing participants to choose whichever of two digit judgments they wanted on each trial (low/high or odd/even). The researchers only requested that participants try to do the tasks in random order. In addition to their main finding of large voluntary task switching costs, they also discovered a strong repetition bias, in which participants switched substantially less than $50 \%$ of the time, which a random sequence would stipulate. This type of switching avoidance was further investigated by Kool and colleagues (e.g., Kool \& Botvinick, 2014), who asked people to choose among sequences of trials that included frequent vs. infrequent task-switching. Participants consistently chose the sequence that entailed less switching, and the researchers established that this choice was motivated by the desire to avoid mental effort.

How do these studies relate to the topic at hand? Attention researchers have shown that frequent updating of one's control state comes with performance costs (e.g., Wolfe, Horowitz, Kenner, Hyle, \& Vasan, 2004), and one can thus speculate that observers might want to avoid such costs when possible. Moreover, a growing number of studies have reported suboptimal use of attentional strategy when participants are able to choose how to perform a visual search task (for reviews, see Clarke, Nowakowska \& Hunt, 2019; Irons \& Leber, 2020). Irons and Leber (2018) produced evidence that participants avoid optimal strategies when such strategies are reported to be subjectively effortful.

To summarize, in asking the question of whether individuals can implement explicit, proactive suppression, we must consider the following points: 1) frequently updating one's control state to suppress changing distractors on a trial-by-trial basis is likely to be costly, 2) task switching is perceived to be effortful, 3) people tend to avoid task switching when possible, 4) attentional strategies that are perceived to be effortful are avoided when possible, 5) participants are not required to attempt explicit proactive control in the experiments seeking to measure it, and 6) researchers may not find evidence of explicit proactive control unless participants implement it uniformly across the entire testing session. Taking all these points together, is it any wonder that we lack consistent evidence for this form of suppression?

\section{Implicit Suppression is Context Dependent}

When considering the alternatives to explicit suppression, the authors - particularly in the Luck and Gaspelin as well as the Theeuwes sections of the article - appeal to a selection history mechanism that emerges after the repeated suppression of a feature or location in space. For instance, Luck and Gaspelin specify that "suppression builds up over a period of several trials with a specific singleton color" (p. 9). Theeuwes describes in multiple places a spatial filtering map whose weights for distractor locations get altered following statistical learning.

While these accounts do not state it explicitly, they seem to imply that implicit suppression is tied to specific features and/or locations. However, implicit suppression is instead arguably context dependent, implemented flexibly from one moment to the next (e.g., switching from one color to another or one location to another).

Unlike low-level perceptual learning, which is long-lasting and tends to be highly specific to properties like shape and retinal location (Goldstone, 1998), there is ample evidence of context dependency in attentional learning. Contextual cueing exemplifies this notion, in which individual spatial locations are quickly prioritized - flexibly from one trial to the next - once the spatial configuration of the search array is revealed (Chun \& Jiang, 1998).

In my work with Howard Egeth, we showed that one's past experience with a feature search or singleton detection mode could dramatically alter whether participants would successfully ignore salient distractors (e.g., Leber \& Egeth, 2006). In subsequent work, Jun Kawahara, Yuji Gabari, and I speculated that the learning effect was specific to the testing room environment. We found support for this idea in an experiment in which participants who were trained to use a specific control state during one session used the same control state in a subsequent test session, up to a week later (Leber, Kawahara \& Gabari, 2009). Assuming participants did not continuously engage in the same control state in the outside world for the entire duration between training and test, we concluded that the testing room environment served to reactivate a learned strategy during the test session. 
Cosman and Vecera (2013) found more direct evidence that learned control states could be tied to specific contexts; they trained subjects to use a singleton detection mode while presented with one category of background scenes (e.g., cities) and a feature search mode while presented with another category of scenes (e.g., forests). Results showed that, during test trials, the background scenes triggered distinct control states (but see Cochrane \& Pratt, 2021, who did not replicate this result). Using a manipulation similar to Cosman and Vecera's, Anderson (2015) found effects of value-driven distractor prioritization to be context specific.

More recent work has shown that space-based distractor suppression is context-dependent and can flexibly change from trial to trial. In a study by Leber, Gwinn, Hong, and O'Toole (2016), we used valid spatial cues to predict the target location, while implicitly cueing the likely distractor location. Critically, each cued target location predicted a distinct distractor location. Results showed that participants flexibly shifted the locations they suppressed from trial to trial, depending on the target cueing context.

More work needs to be done to determine whether context-dependent suppression is qualitatively similar to other reports of suppression (e.g., is it proactive or reactive?). Nevertheless, the existence of context-dependent suppression shows that experience-driven attention, or selection history, need not operate on low-level stimulus properties (e.g., the cumulative building of suppression at a single location in an attentional priority map). Rather, the control states of the observer are activated by environmental triggers, based on associative links between context and task (see, e.g., the Attention to Action model of Norman \& Shallice, 1986).

The theoretical weight of context dependency is substantial, as it determines how and from where suppression signals are generated. It is therefore difficult to imagine any successful mechanistic explanation of suppression that is agnostic to context. It is incumbent upon us to specify our models accordingly.

\section{Summary and Conclusion}

I have addressed two related points about suppression and attentional control. First, while there exists much skepticism about whether people can use explicit, proactive suppression, I argue that we have not given our participants a fair chance to demonstrate their full range of abilities. New, clever approaches promise to help resolve this question. Second, experience-driven attention, or selection history, is more high-level and adaptive than is oft acknowledged, and any complete model must reflect this.

Nevertheless, we do not know the full extent of context dependency in attentional learning, and more studies are prudent.

In conclusion, I appreciate the authors' efforts to find common ground and outline significant outstanding questions about attentional capture and control. Their article promises to be a pivotal document in charting a path forward for years to come.

\section{Acknowledgment}

I thank Alon Zivony for helpful comments and suggestions. Support provided by NSF BCS-2021038 to A. B. L.

\section{References}

Anderson, B. A. (2015). Value-driven attentional priority is context specific. Psychonomic Bulletin \& Review, 22(3), 750-756.

Arrington, C. M., \& Logan, G. D. (2004). The cost of a voluntary task switch. Psychological Science, 15(9), 610-615.

Bacon, W. F., \& Egeth, H. E. (1994). Overriding stimulus-driven attentional capture. Perception \& psychophysics, 55(5), 485-496.

Chun, M. M., \& Jiang, Y. (1998). Contextual cueing: Implicit learning and memory of visual context guides spatial attention. Cognitive psychology, 36(1), 28-71.

Clarke, A. D., Nowakowska, A., \& Hunt, A. R. (2019). Seeing beyond salience and guidance: the role of bias and decision in visual search. Vision, 3(3), 46.

Cochrane, B. A., \& Pratt, J. (2021). Context isn't everything: Search performance is influenced by the nature of the task but not the background. Attention, Perception, \& Psychophysics, 83(1), 27-37. 
Cosman, J. D., \& Vecera, S. P. (2013). Context-dependent control over attentional capture. Journal of Experimental Psychology: Human Perception and Performance, 39(3), 836.

Egeth, H. E., Leonard, C. J., \& Leber, A. B. (2010). Why salience is not enough: Reflections on top-down selection in vision. Acta Psychologica, 135(2), 130.

Goldstone, R. L. (1998). Perceptual learning. Annual review of psychology, 49(1), 585-612.

Irons, J. L., \& Leber, A. B. (2016). Choosing attentional control settings in a dynamically changing environment. Attention, Perception, \& Psychophysics, 78(7), 2031-2048.

Irons, J. L., \& Leber, A. B. (2020). Developing an individual profile of attentional control strategy. Current Directions in Psychological Science, 29(4), 364-371.

Kool, W., \& Botvinick, M. (2014). A labor/leisure tradeoff in cognitive control. Journal of Experimental Psychology: General, 143(1), 131.

Leber, A. B., \& Egeth, H. E. (2006). Attention on autopilot: Past experience and attentional set. Visual Cognition, 14(4-8), 565-583.

Leber, A. B., Gwinn, R. E., Hong, Y., \& O’Toole, R. J. (2016). Implicitly learned suppression of irrelevant spatial locations. Psychonomic Bulletin \& Review, 23(6), 1873-1881.

Leber, A. B., Kawahara, J. I., \& Gabari, Y. (2009). Long-term abstract learning of attentional set. Journal of Experimental Psychology: Human Perception and Performance, 35(5), 1385.

Luck, S. J., Gaspelin, N., Folk, C. L., Remington, R. W., \& Theeuwes, J. (2021). Progress toward resolving the attentional capture debate. Visual Cognition, 29(1), 1-21.

Monsell, S. (2003). Task switching. Trends in cognitive sciences, 7(3), 134-140.

Norman, D. A., \& Shallice, T. (1986). Attention to action: Willed and automatic control of behavior. In R. J. Davidson, G. E. Schwartz, \& D. Shapiro (Eds.), Advances in research: Vol. IV. Consciousness and self-regulation (pp. 1-18). New York: Plenum Press.

Wolfe, J. M., Horowitz, T. S., Kenner, N., Hyle, M., \& Vasan, N. (2004). How fast can you change your mind? The speed of top-down guidance in visual search. Vision research, 44(12), 1411-1426. 